\title{
Deformaciones de Haces Cilíndricos y Cónicos al atravesar una Placa Plano-Paralela de Cristal Uniaxial
}

\author{
Deformations of Cylindrical and Conical Beams Transmitted through a Uniaxial Crystal \\ Parallel-Plane Plate \\ Francisco E. Veiras ${ }^{\# * 1}$, Liliana I. Perez ${ }^{\# \& 2}$ \\ ${ }^{\#}$ Grupo de Láser, Óptica de Materiales y Aplicaciones Electromagnéticas, Departamento de Física, Facultad de Ingeniería, \\ Universidad de Buenos Aires \\ Av. Paseo Colón 850 - (C1063ACV) CABA - Argentina \\ * Consejo Nacional de Investigaciones Científicas y Técnicas \\ ${ }^{\&}$ Universidad de Buenos Aires. Consejo Nacional de Investigaciones Científicas y Técnicas. Instituto de Tecnologías y \\ Ciencias de la Ingeniería "Hilario Fernández Long". Facultad de Ingeniería. \\ 1 fveiras@fi.uba.r \\ 2 lperez@fi.uba.ar
}

Recibido: 17/05/18; Aceptado: 09/06/18

\begin{abstract}
One of the most used tools in optics is ray tracing, due to the fact that is a fundamental tool for optic instruments design. In this work we analyze the successive deformations that both cylindrical (first order approximation of a collimated beam) and conical (first order approximation of a converging or divergent beam) beams suffer when they impinge normal to a uniaxial crystal plane-parallel plate and go through the different interfaces. This is accomplished by following the path of each incident ray on the first interface. The ray tracing for the ordinary beam is the same as in isotropic media, but the extraordinary beam ray tracing is more difficult, for these rays are no longer contained in the incidence plane and their directions are different from those of the corresponding normals to the wavefront. We also analyze the loss of revolution symmetry of the beams and the image formation.
\end{abstract}

Keywords: anisotropic optical materials; image formation theory; integrated optics components.

Resumen- Una de las herramientas más usadas en la óptica es el trazado de rayos ya que es la herramienta fundamental para el diseño de todo instrumento óptico. En este trabajo se analizan las sucesivas deformaciones que un haz cilíndrico (modelo a primer orden de un haz colimado) y un haz cónico (modelo a primer orden de un haz divergente o convergente), que inciden normalmente sobre una placa planoparalela de cristal uniaxial, sufren a medida que van atravesando las distintas interfaces. Esto se hace siguiendo el camino de cada rayo incidente sobre la primera interfaz. El trazado de rayos para los rayos ordinarios es igual al trazado de rayos en medios isótropos. El trazado de rayos extraordinario, en cambio, presenta más dificultad ya que los rayos no están contenidos en el plano de incidencia y no coinciden con las normales a los frentes de onda. Se analizan también la pérdida de simetría de revolución de los haces y la formación de las sucesivas imágenes.

Palabras clave: materiales ópticos anisótropos; teoría de formación de imágenes; componentes para óptica integrada.

\section{INTRODUCCIÓN}

Los fenómenos ópticos que pueden observarse a través de una placa plano-paralela con anisotropía eléctrica son de una sorprendente multiplicidad: doble imagen en un cristal de calcita naturalmente clivado (birrefringencia), desaparición de una de las imágenes al girar la placa si se miran a través de un polarizador (polarización), cambio del estado de polarización de la luz al atravesarla (retardo de fase), producción de figuras de interferencia, etc. A pesar de ser conocidos desde el siglo XVII [1] el estudio de las propiedades de este tipo de medios sigue planteando una imprevisible cantidad de interrogantes y cada día aparecen nuevos recursos para posibles aplicaciones tecnológicas.

Si una onda plana incide sobre la superficie de separación entre un medio isótropo y otro anisótropo, existirán, en general, una onda reflejada y dos ondas transmitidas. Para determinados tipos de medios anisótropos, llamados uniaxiales, las ondas transmitidas son denominadas onda ordinaria y onda extraordinaria $\mathrm{y}$, en general, tienen distintas direcciones $\mathrm{y}$ velocidades de propagación. Este comportamiento se puede explicar considerando que un medio uniaxial tiene dos índices de refracción (llamados índices de refracción principales ordinario $n_{o} \mathrm{y}$ extraordinario $n_{e}$ ) y una dirección preferencial en el espacio, denominada eje óptico $\breve{z}_{3}$. La velocidad 
de fase de la onda ordinaria (que da lugar al índice de refracción principal ordinario) resulta independiente de la dirección de propagación, En cambio, la velocidad de fase de la onda extraordinaria (que da lugar a un índice de refracción extraordinario) depende no sólo de los índices principales $n_{o}$ y $n_{e}$ sino también de la dirección de propagación de la onda respecto al eje óptico. A pesar de estas particularidades es posible encontrar una expresión para la Ley de Snell tanto para la refracción ordinaria (idéntica a la de medios isótropos) como para la refracción extraordinaria. En este último caso, la expresión resultante tiene la particularidad de que tanto el índice de refracción extraordinario como el ángulo de refracción dependen de los índices principales y de la dirección del eje óptico. Esta particularidad lleva a que la dirección de propagación de la onda extraordinaria no coincida con la dirección de avance de la energía asociada (Fig.1). Para interfaces formadas por un medio uniaxial y uno isótropo, se tienen en cuenta las mismas consideraciones [2,3].

En varios trabajos anteriores se han abordado problemas relacionados con la formación de imágenes [2-6, 7], con el camino óptico [8,9] y las figuras de interferencia [10,11] para distintos cortes del cristal. En los últimos años hemos obtenido expresiones analíticas generales $[12,13]$ que han permitido el desarrollo de polarímetros solares [14] e interferómetros para diagnóstico de plasma $[15,16]$. Asimismo, los estudios de patrones de interferencia conoscópicos [17] nos han permitido desarrollar moduladores $\mathrm{y}$ demoduladores de fase birrefringentes $[18,19$, 20].

Cuando se consideran modelos de haces de luz monocromáticos y limitados en el espacio como superposición de infinitas ondas planas (componentes de Fourier escalares o vectoriales), aparecen distintos efectos en la propagación, reflexión y transmisión en medios isótropos o anisótropos, lineales o no lineales, dieléctricos o absorbentes. Sin embargo, en todos los casos, los efectos de primer orden se corresponden con los obtenidos a partir de los modelos de óptica geométrica (considerándolos manojos de rayos más o menos complejos) [12].

Aquí presentamos un análisis de las deformaciones que sufren determinados manojos de rayos, cuando atraviesan una placa plano- paralela de cristal uniaxial, teniendo en cuenta sólo el trazado de rayos.

En primer lugar se presentan en forma resumida las fórmulas necesarias para calcular las direcciones de los rayos refractados en las interfaces que delimitan las placas plano-paralelas construidas con materiales dieléctricos uniaxiales. Luego se muestran y analizan las deformaciones sufridas por dos tipos de haces tridimensionales sobre estos tipos de placas cuando la dirección de onda media es normal a la placa. Primero se trata el caso más sencillo que corresponde a un haz cilíndrico (que corresponde al modelado de un haz colimado) y, luego a un haz cónico cuyo punto de convergencia (real o imaginario) es totalmente arbitrario (modelado de un haz proveniente de una fuente puntual o de una lente objetivo).

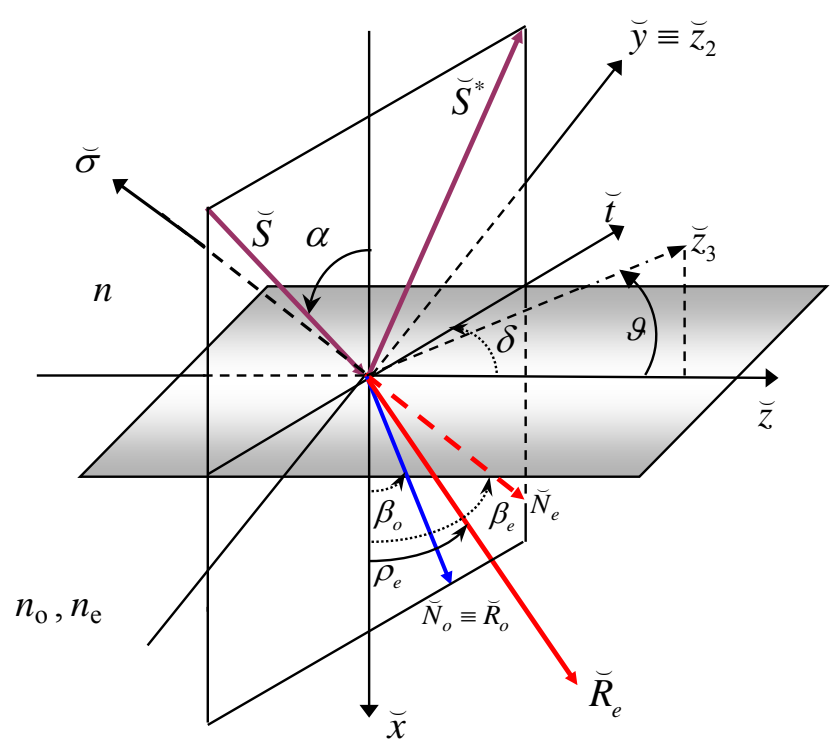

(a)

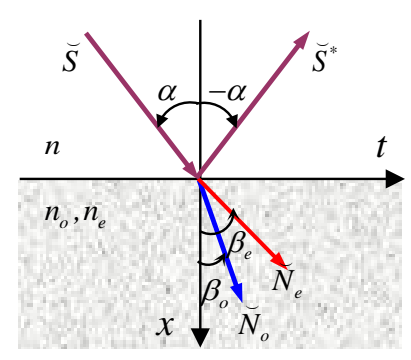

(b)

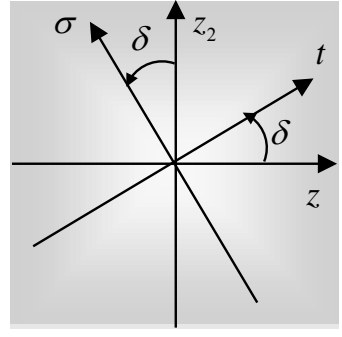

(c)
Fig.1. Sistemas de coordenadas. (a) Interfaz medio isótropo-cristal uniaxial. El eje óptico $\breve{Z}_{3}$ forma un ángulo $\vartheta$ con la interfaz y un ángulo $\delta$ con el plano de incidencia. (b) Plano de incidencia (c) Plano que corresponde a una interfaz. 


\section{LA TRANSMISIÓN A TRAVÉS DE UNA PLACA PLANO- PARALELA UNIAXIAL}

La refracción de un rayo de luz que incide en una placa plano-paralela birrefringente uniaxial, inmersa en un único medio isótropo de índice de refracción $n$, se calcula teniendo en cuenta las dos interfaces que limitan la placa [21]. En la primera interfaz, el rayo de luz (de dirección $\breve{S}$ ) incide desde el medio isótropo sobre el cristal uniaxial y da lugar a dos rayos refractados de direcciones $\breve{R}_{o}$ (rayo ordinario) y $\breve{R}_{e}$ (rayo extraordinario) (Fig. 1). En la segunda interfaz estos rayos de luz inciden desde el cristal y dan lugar en el medio isótropo a los rayos $\breve{S}_{o}$ (rayo proveniente del rayo ordinario) y $\breve{S}_{e} \quad$ (rayo proveniente del rayo extraordinario) $[2,3,21,23]$ (Fig. 2). En ella puede verse en detalle cómo son los corrimientos en cada una de las superficies: la superficie de salida de la placa y una arbitraria a una distancia $D$ de ella.

Para escribir las ecuaciones que dan las direcciones y posiciones de estos rayos en función de los parámetros característicos de la placa (índices de refracción principales $n_{o}, n_{e} \mathrm{y}$ dirección del eje óptico $\breve{z}_{3}$ ) y de la dirección y posición del rayo incidente, se define el versor $\breve{X}$ (perpendicular a la placa), el versor $\breve{t}$ (perpendicular a $\breve{x}$ y contenido en el plano de incidencia) y el versor $\breve{\sigma}$ (perpendicular a los otros dos versores) (Fig. 2). De modo que el versor en la dirección del eje óptico es

$$
\begin{aligned}
& \breve{z}_{3}=\breve{x}\left(\breve{z}_{3} \cdot \breve{x}\right)+\breve{\sigma}\left(\breve{z}_{3} \cdot \breve{\sigma}\right)+\breve{t}\left(\breve{z}_{3} \cdot \breve{t}\right) \\
& \breve{z}_{3}=-\breve{x} \sin \vartheta-\breve{\sigma} \cos \vartheta \sin \delta+\breve{t} \cos \vartheta \cos \delta
\end{aligned}
$$

donde $\vartheta$ es el ángulo que forma el eje óptico con la interfaz y $\delta$ es el ángulo que forma el plano de incidencia con el plano que contiene al eje óptico y la normal a las interfaces (Fig. 1(c)) [2]. Además, definimos arbitrariamente al eje $z$ como la proyección del eje óptico sobre la interfaz. Es decir,

$$
\begin{aligned}
& \breve{z}_{3}=\breve{x}\left(\breve{z}_{3} \cdot \breve{x}\right)+\breve{z} \quad\left(\breve{z}_{3} \cdot \breve{z}\right) \\
& \breve{z}_{3}=-\breve{x} \sin \vartheta+\breve{z} \cos \vartheta
\end{aligned}
$$

El plano de incidencia queda así definido por el versor $\breve{x}$ y la dirección del rayo incidente $\breve{S}$, donde

$$
\begin{aligned}
& \breve{S}=\breve{x}(\breve{S} . \breve{x})+\breve{t}(\breve{S} . \breve{t}) \\
& \breve{S}=\breve{x} \cos \alpha+\breve{t} \sin \alpha
\end{aligned}
$$

siendo $\alpha$ el ángulo de incidencia y estando el origen de coordenadas en el punto de incidencia sobre la primera interfaz (Fig.1(a))
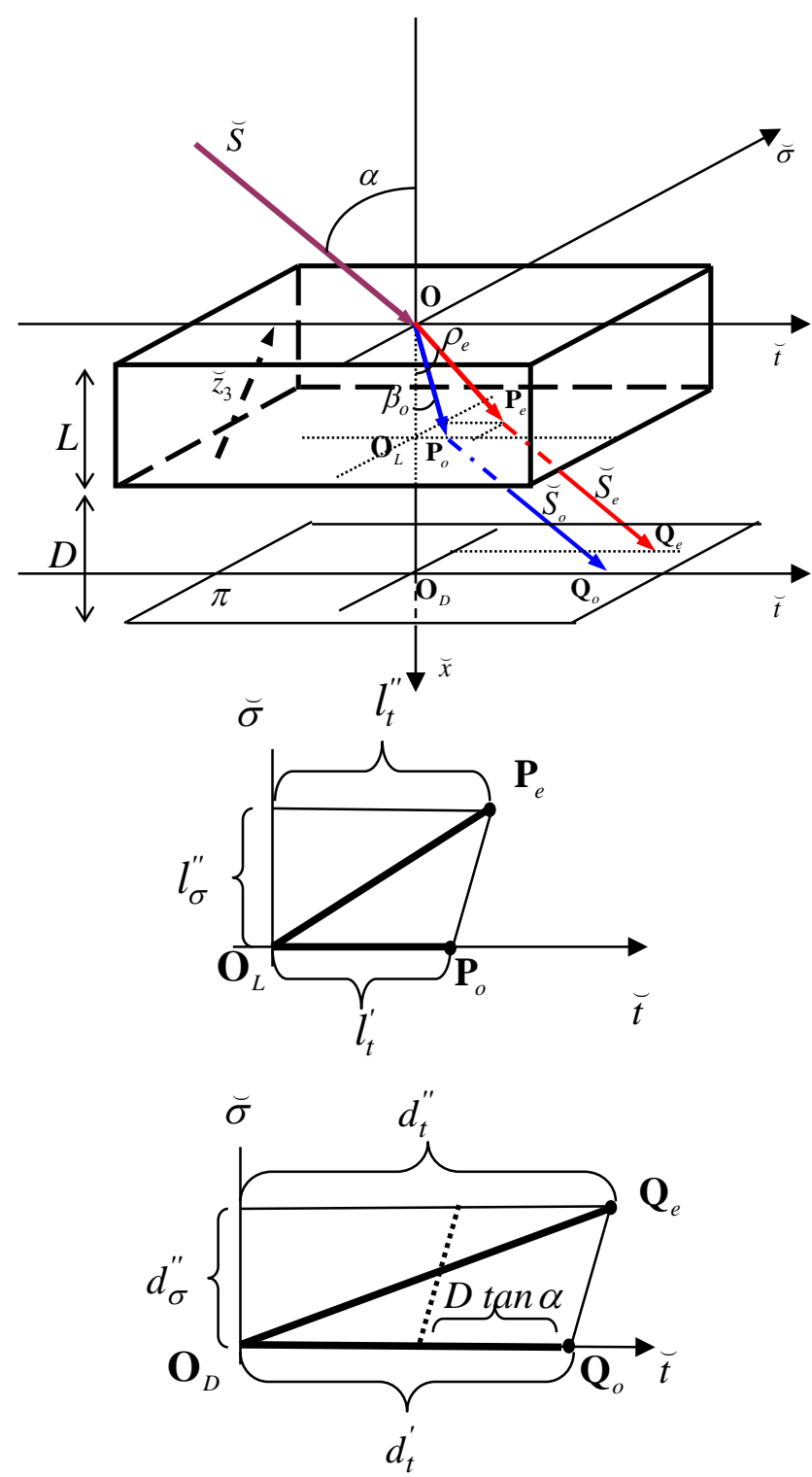

Fig.2. Transmisión de un rayo a través de una placa plano-paralela uniaxial. Abajo, detalles de los corrimientos ordinario y extraordinario sobre la segunda interfaz y sobre un plano $\Pi$ que se encuentra a una distancia $D$ de la placa.

\section{A. Transmisión ordinaria}

En una interfaz formada por un medio isótropo y un cristal uniaxial, la dirección del rayo refractado ordinario coincide con la normal al frente de ondas $\breve{R}_{o}=\breve{N}_{o}$

$$
\breve{N}_{o}=\breve{x}\left(\breve{N}_{o} \cdot \breve{x}\right)+\breve{t}\left(\breve{N}_{o} \cdot \breve{t}\right)
$$

donde $\breve{N}_{o}$ resulta de la Ley de Snell

$$
n(\breve{S} \cdot \breve{t})=n_{o}\left(\breve{N}_{o} \cdot \breve{t}\right)
$$

El punto de incidencia sobre la segunda interfaz estará desplazado con respecto al punto de incidencia de la primera interfaz (Fig. 3b)) en: 


$$
l_{t}^{\prime}=L \frac{n \sin \alpha}{\left(n_{o}^{2}-n^{2} \sin ^{2} \alpha\right)^{1 / 2}} \quad l_{\sigma}^{\prime}=0
$$

donde $L$ es el espesor de la placa (Fig.2(a) y (b)). Denotando con $l_{y}^{\prime}$ y $l_{z}^{\prime}$ a las coordenadas y y $z$ del punto de incidencia sobre la segunda interfaz, y haciendo la rotación de coordenadas correspondiente (Fig.1(c)) se obtienen los desplazamientos sobre la superficie inferior de la placa

$$
\begin{aligned}
& l_{y}^{\prime}=L \frac{n \sin \alpha}{\left(n_{o}^{2}-n^{2} \sin ^{2} \alpha\right)^{1 / 2}} \sin \delta \\
& l_{z}^{\prime}=L \frac{n \sin \alpha}{\left(n_{o}^{2}-n^{2} \sin ^{2} \alpha\right)^{1 / 2}} \cos \delta
\end{aligned}
$$

El rayo ordinario se refracta en la segunda interfaz dando lugar a uno de los rayos transmitidos

$$
\breve{S}_{o}=\breve{x}\left(\breve{S}_{o} \cdot \breve{x}\right)+\breve{t}\left(\breve{S}_{o} . \breve{t}\right)
$$

que tiene la misma dirección que el rayo incidente $\breve{S}$ pero está desplazado con respecto a éste en $l_{t}^{\prime}$. En un plano paralelo a la placa a una distancia $D$ de la segunda interfaz (plano $\Pi$ de la Fig.2), el punto de intersección del rayo ordinario con dicho plano $\Pi$ estará a una distancia del eje $x$ dada por

$$
d_{t}^{\prime}=l_{t}^{\prime}+D \tan \alpha
$$
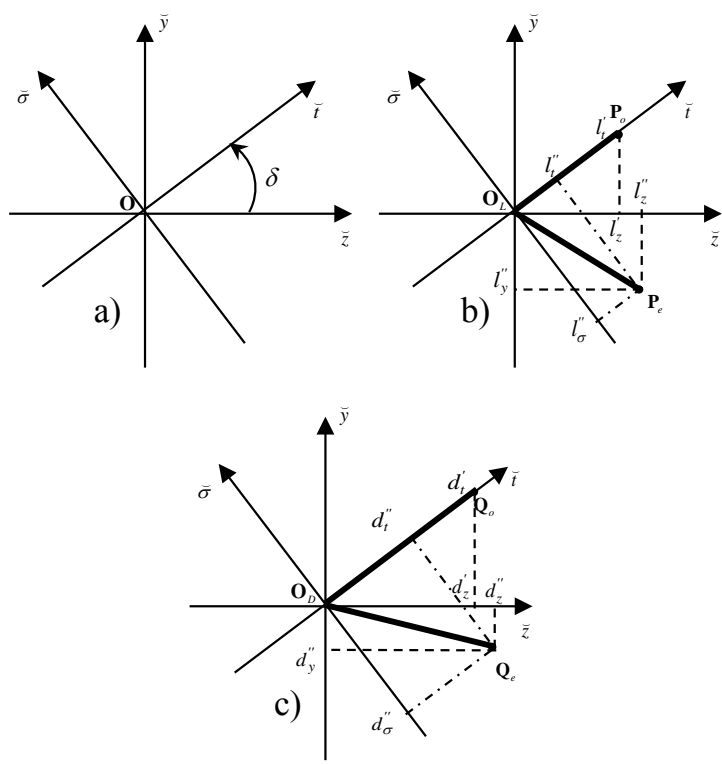

Fig.3. Sistemas de coordenadas y corrimientos sobre los tres planos característicos. (a) superficie superior de la placa (interfaz isótropouniaxial) (b) superficie inferior de la placa (interfaz uniaxial-isótropo) (c) un plano $\Pi$

Es decir que las coordenadas y y $z$ del punto de incidencia del rayo ordinario sobre el plano $\Pi$ son (Fig.3c))

$$
\begin{aligned}
& d_{y}^{\prime}=\left(L \frac{n \sin \alpha}{\left(n_{o}^{2}-n^{2} \sin ^{2} \alpha\right)^{1 / 2}}+D \tan \alpha\right) \cos \delta \\
& d_{z}^{\prime}=\left(L \frac{n \sin \alpha}{\left(n_{o}^{2}-n^{2} \sin ^{2} \alpha\right)^{1 / 2}}+D \tan \alpha\right) \sin \delta
\end{aligned}
$$

como resulta de las ecs. (7) y (9).

\section{B. Transmisión extraordinaria}

El cálculo de la dirección del rayo refractado extraordinario es bastante más complicado y puede encontrarse un desarrollo detallado en trabajos anteriores $[2,3,21,22]$. Aquí transcribimos las fórmulas necesarias para los cálculos posteriores. Para abreviar la escritura de las ecuaciones que resultan bastante extensas, se definieron los siguientes parámetros auxiliares [22]

$$
\begin{aligned}
h_{x} & =n_{o}^{2}+\left(n_{e}^{2}-n_{o}^{2}\right)\left(\breve{z}_{3} \cdot \breve{x}\right)^{2} \\
h_{\sigma} & =n_{e}^{2}-\left(n_{e}^{2}-n_{o}^{2}\right)\left(\breve{z}_{3} \cdot \breve{\sigma}\right)^{2} \\
h_{t} & =n_{o}^{2}+\left(n_{e}^{2}-n_{o}^{2}\right)\left(\breve{z}_{3} \cdot \breve{t}\right)^{2} \\
h_{x t} & =\left(n_{e}^{2}-n_{o}^{2}\right)\left(\breve{z}_{3} \cdot \breve{x}\right)\left(\breve{z}_{3} \cdot \breve{t}\right) \\
h_{x \sigma} & =\left(n_{e}^{2}-n_{o}^{2}\right)\left(\breve{z}_{3} \cdot \breve{x}\right)\left(\breve{z}_{3} \cdot \breve{\sigma}\right) \\
h_{\sigma t} & =\left(n_{e}^{2}-n_{o}^{2}\right)\left(\breve{z}_{3} \cdot \breve{\sigma}\right)\left(\breve{z}_{3} \cdot \breve{t}\right)
\end{aligned}
$$

La dirección del rayo refractado extraordinario se obtiene calculando primero la dirección de la normal al frente de ondas $\breve{N}_{e}$ que está contenida en el plano de incidencia, es decir,

$$
\breve{N}_{e}=\breve{x}\left(\breve{N}_{e} \cdot \widetilde{x}\right)+\breve{t}\left(\breve{N}_{e} \cdot \breve{t}\right)
$$

donde para $\left(\breve{N}_{e} . \breve{t}\right)$ vale una Ley de Snell generalizada con un índice de refracción $n^{\prime \prime}$ que depende de la dirección de incidencia

$$
n(S \cdot \breve{t})=n^{\prime \prime}\left(\breve{N}_{e} \cdot \breve{t}\right)
$$

y $n^{\prime \prime}$ está dado por [21]

$$
n^{\prime \prime 2}=\frac{1}{h_{x}^{2}}\left(\left(n_{o} \Delta^{1 / 2}-h_{x t} n \sin \alpha\right)^{2}+h_{x}^{2} n^{2} \sin ^{2} \alpha\right)
$$

con

$$
\Delta=n_{e}^{2} h_{x}-h_{\sigma} n^{2} \sin ^{2} \alpha
$$

Obtenida la dirección de $\breve{N}_{e}$, puede calcularse la dirección del rayo $\breve{R}_{e}$ mediante la relación vectorial $[2,20]$

$$
\begin{gathered}
\breve{R}_{e}=\frac{1}{f_{e}}\left(n_{o}{ }^{2} \breve{N}_{e}+\left(n_{e}^{2}-n_{o}^{2}\right)\left(\breve{N}_{e} \cdot \breve{z}_{3}\right) \breve{Z}_{3}\right) \\
f_{e}^{2}=n_{o}^{4}+\left(n_{e}^{4}-n_{o}^{4}\right)\left(N_{e} z_{3}\right)^{2}
\end{gathered}
$$


Las componentes del versor $\breve{R}_{e}$ que resultan son

$$
\begin{aligned}
& \left(\breve{R}_{e} \cdot \breve{x}\right)=\frac{n_{o} h_{x} \Delta^{1 / 2}}{n^{\prime \prime} f_{e} h_{x}} \\
& \left(\breve{R}_{e} \cdot \breve{\sigma}\right)=\frac{n_{o}\left(h_{x \sigma} \Delta^{1 / 2}+n_{o} h_{\sigma t} n(\breve{S} \cdot \breve{t})\right)}{n^{\prime \prime} f_{e} h_{x}} \\
& \left(\breve{R_{e}} \cdot \breve{t}\right)=\frac{n_{o}\left(h_{x t} \Delta^{1 / 2}+n_{o} h_{\sigma} n(\breve{S} \cdot \breve{t})\right)}{n^{\prime \prime} f_{e} h_{x}}
\end{aligned}
$$

Para la refracción en la segunda interfaz, vale la Ley de Snell generalizada que toma la forma

$$
n^{\prime \prime}\left(\breve{N}_{e} \cdot \breve{t}\right)=n\left(S_{e} \cdot \breve{t}\right)
$$

de donde resulta, como era de esperar, que el rayo extraordinario que sale de la placa es paralelo al rayo incidente y al rayo ordinario. La posición de este rayo, sin embargo, está dada por el punto de incidencia del rayo extraordinario sobre la segunda interfaz cuyas coordenadas $\sigma$ y $t$ estarán dadas por

$$
l_{\sigma}^{\prime \prime}=L \frac{\left(\breve{R}_{e} \cdot \breve{\sigma}\right)}{\left(\breve{R}_{e} \cdot \bar{x}\right)} \quad l_{t}^{\prime \prime}=L \frac{\left(\breve{R}_{e} \cdot \breve{t}\right)}{\left(\breve{R}_{e} \cdot \bar{x}\right)}
$$

Sus coordenadas y y $z$ resultan de la rotación de coordenadas (Fig.3)

$$
\begin{aligned}
& l_{y}^{\prime \prime}=l_{t}^{\prime \prime} \sin \delta+l_{\sigma}^{\prime \prime} \cos \delta \\
& l_{z}^{\prime \prime}=l_{t}^{\prime \prime} \cos \delta-l_{\sigma}^{\prime \prime} \sin \delta
\end{aligned}
$$

Reemplazando ecs. (23), (24) y (25) en (27) y (28) se obtiene

$$
\begin{gathered}
l_{y}^{\prime \prime}=L \frac{n_{o} n \sin \alpha \sin \delta}{\left(n_{e}^{2} h_{x}-h_{\sigma} n^{2} \sin ^{2} \alpha\right)^{1 / 2}} \\
l_{z}^{\prime \prime}=L \frac{n_{e}^{2} n_{o} n \sin \alpha \cos \delta}{h_{x}\left(n_{e}^{2} h_{x}-h_{\sigma} n^{2} \sin ^{2} \alpha\right)^{1 / 2}}
\end{gathered}
$$

con

$$
A=-L \frac{\left(n_{e}^{2}-n_{o}^{2}\right) \sin \vartheta \cos \vartheta}{h_{x}}
$$

Es decir, que para un rayo que incide perpendicularmente a la placa, el rayo refractado se desvía de la normal con un ángulo $\eta_{e}$ cuya tangente está dada por

$$
\tan \eta_{e}=A=-\frac{\left(n_{e}^{2}-n_{o}^{2}\right) \sin \vartheta \cos \vartheta}{n_{o}^{2} \cos ^{2} \vartheta+n_{e}^{2} \sin ^{2} \vartheta}
$$

De esta manera, el punto de incidencia sobre la segunda interfaz estará dado por

$$
\left.l_{y}^{\prime \prime}\right|_{\alpha=0}=\left.0 \quad l_{z}^{\prime \prime}\right|_{\alpha=0}=A
$$

Una vez transmitido el haz a través de la placa, las coordenadas del punto de incidencia del rayo extraordinario sobre el plano $\Pi$ pueden determinarse a partir de

$$
\begin{aligned}
& d_{y}^{\prime \prime}=l_{y}^{\prime \prime}+D \tan \alpha \sin \delta \\
& d_{z}^{\prime \prime}=l_{z}^{\prime \prime}+D \tan \alpha \cos \delta
\end{aligned}
$$

A partir de las ecs. (29), (30) y (34) las expresiones para dichas coordenadas resultan

$$
\begin{gathered}
d_{y}^{\prime \prime}=\left(L \frac{n_{o} n \sin \alpha}{\left(n_{e}^{2} h_{x}-h_{\sigma} n^{2} \sin ^{2} \alpha\right)^{1 / 2}}+D \tan \alpha\right) \sin \delta \\
d_{z}^{\prime \prime}=\left(L \frac{n_{e}^{2} n_{o} n \sin \alpha}{h_{x}\left(n_{e}^{2} h_{x}-h_{\sigma} n^{2} \sin ^{2} \alpha\right)^{1 / 2}}+D \tan \alpha\right) \cos \delta+A(
\end{gathered}
$$

\section{TRANSMISIÓN DE UN HAZ CILÍNDRICO CON INCIDENCIA NORMAL}

Consideremos un conjunto de rayos dispuestos sobre una superficie cilíndrica de radio $r$ que incide en forma perpendicular sobre la placa (Fig. 4). Las coordenadas de los puntos de incidencia en la primera interfaz están dadas por

$$
\begin{aligned}
& l_{y}=r \sin \delta \\
& l_{z}=r \cos \delta
\end{aligned}
$$

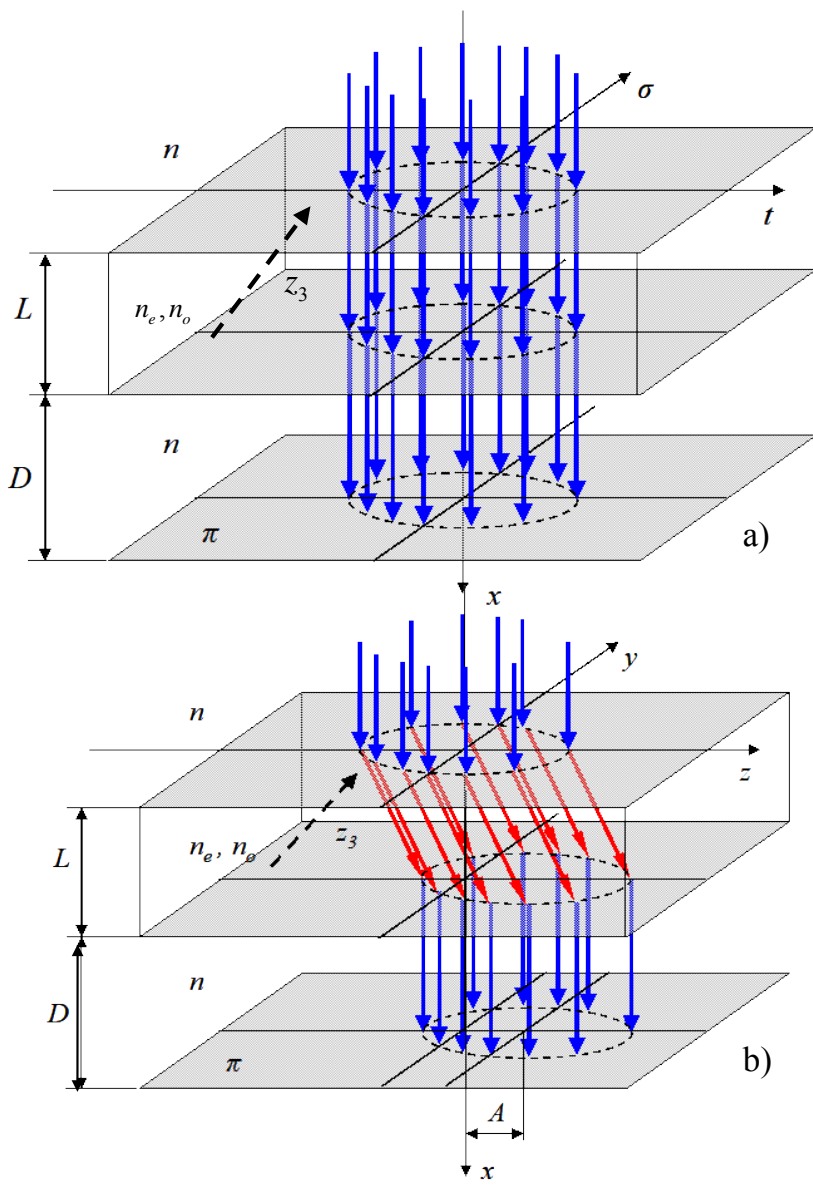

Fig.4. Transmisión de un haz cilíndrico a través de una placa plano-paralela uniaxial. Sobre el plano $\pi$ se observan los haces provenientes del haz ordinario a) y del extraordinario b)

Como para incidencia perpendicular a la placa, los rayos ordinarios no se desvían, las coordenadas de los puntos de incidencia sobre la 
segunda interfaz y sobre el plano $\Pi$ serán las mismas que las dadas por la ec.(37), es decir,

$$
\begin{aligned}
& l_{y}^{\prime}=d_{y}^{\prime}=r \sin \delta \\
& l_{z}^{\prime}=d_{z}^{\prime}=r \cos \delta
\end{aligned}
$$

Además, como esto vale para cualquier radio $r$, tendremos que un haz cilíndrico que incide perpendicularmente a la placa da lugar a un haz trasmitido ordinario de radio $r_{o}=r$.

Los rayos extraordinarios, en cambio, sufren una desviación en la dirección de la proyección del eje óptico, que para $\alpha=0$ está dada por las ecs. (31) y (32). De éstas resulta que las coordenadas de los puntos de incidencia sobre la segunda interfaz están dadas por

$$
\begin{aligned}
& l_{y}^{\prime \prime}=r \sin \delta \\
& l_{z}^{\prime \prime}=r \cos \delta+A
\end{aligned}
$$

De la ec.(39) vemos que los puntos de incidencia sobre la cara inferior de la placa están sobre una circunferencia de radio $r_{e}$ y con el centro desplazado en $A$, es decir,

$$
r_{e}^{2}=l_{y}^{\prime 2}+\left(l_{z}^{\prime \prime}-A\right)^{2}
$$

Los rayos refractados en la cara inferior son perpendiculares a la placa y los puntos de incidencia en el plano $\Pi$ tienen las mismas coordenadas dadas en ec.(39) independientemente de la distancia de la placa al plano, es decir

$$
\begin{aligned}
& d_{y}^{\prime \prime}=r \sin \delta \\
& d_{z}^{\prime \prime}=r \cos \delta+A
\end{aligned}
$$

Por lo tanto vemos que un haz cilíndrico que incida perpendicular a la placa da lugar a dos haces refractados en el interior de la placa: el ordinario que tiene la misma forma, dirección y posición que el incidente; y el extraordinario que está inclinado un ángulo $\eta_{e}$ con respecto a la normal a la placa y cuya intersección con la segunda interfaz es una circunferencia de radio igual al del haz incidente. Este haz refractado resulta en el interior de la placa de forma elíptica pues la intersección del haz con un plano perpendicular al haz es una elipse de eje mayor $a=r / \cos \eta_{e}$ y de eje menor $b=r$. A la salida de la placa se obtienen dos haces perpendiculares a la placa de la misma forma y tamaño que el haz incidente pero separados en una distancia $A$ dada por ec.(31) que depende de los índices de refracción principales del cristal $n_{o}$ y $n_{e}$, y de la dirección del eje óptico de la placa. En las Fig. 5 se muestran los diagramas de los puntos de incidencia en las dos caras de la placa y en un plano $\Pi$ para un cristal negativo (calcita) y para otro positivo (rutilo) con la misma dirección del eje óptico $\left(\vartheta=40^{\circ}\right)$. Se consideraron haces cilíndricos de $r=0.5 \mathrm{~mm}$ incidentes sobre placas de espesor $L=1 \mathrm{~cm}$, siendo la longitud de onda en el vacío $\lambda=632.8 \mathrm{~nm}$.
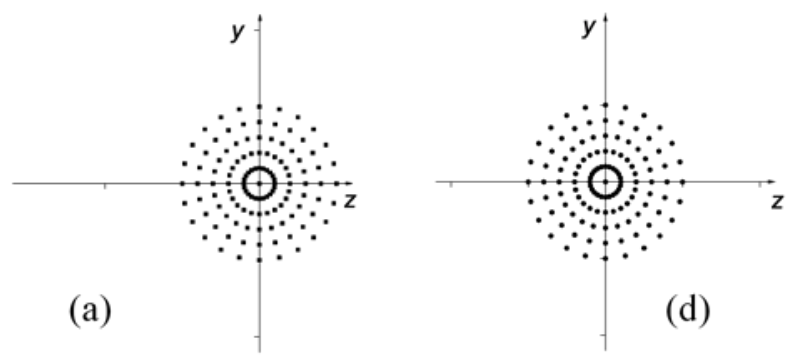

(d)
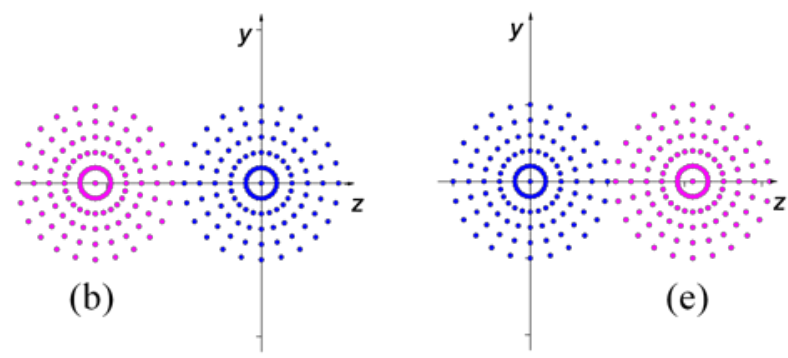

(e)

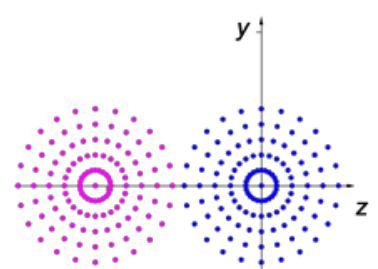

(c)

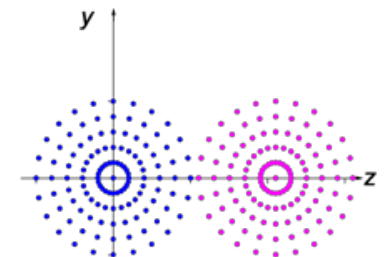

(f)

Figure 5: Diagramas sobre los tres planos característicos para una placa de calcita (izquierda) y para una de rutilo (derecha). (a) y (d) corresponden a la cara superior, (b) y (e) a la cara inferior y (c) y (f) a cualquier plano П. Los tamaños de los haces ordinario (en azul) y extraordinario (magenta) no cambian.

\section{TRANSMISIÓN DE UN HAZ CÓNICO}

Cuando un haz cónico de apertura $\alpha_{0}$ incide en la placa de modo que su dirección media es normal a la placa, cada rayo incidente varía su punto de incidencia, y el ángulo de incidencia varía entre 0 y $\alpha_{0}$. Si consideramos un manojo de rayos dispuesto sobre una superficie cónica, el ángulo de incidencia $\alpha$ será el mismo para todos los rayos y los puntos de incidencia estarán dados por

$$
\begin{aligned}
& l_{y}=\overline{O P_{C}} \tan \alpha \sin \delta \\
& l_{y}=\overline{O P_{C}} \tan \alpha \cos \delta
\end{aligned}
$$


donde $\overline{O P_{C}}$ es la distancia del punto de convergencia del haz a la primera interfaz (Fig.6).

Los rayos ordinarios se refractan según la ec.(5) y los puntos de incidencia sobre la segunda interfaz están sobre una circunferencia de radio $r$ '. Teniendo en cuenta las ecs. (7) y (42), resulta

$$
r^{\prime}=L \frac{n \sin \alpha}{\left(n_{o}^{2}-n^{2} \sin ^{2} \alpha\right)^{1 / 2}}-\overline{O P_{C}} \tan \alpha
$$

de modo que las coordenadas $l_{y}^{\prime}$ y $l_{z}^{\prime}$ son

$$
\begin{aligned}
& l_{y}^{\prime}=r^{\prime} \sin \delta \\
& l_{z}^{\prime}=r^{\prime} \cos \delta
\end{aligned}
$$

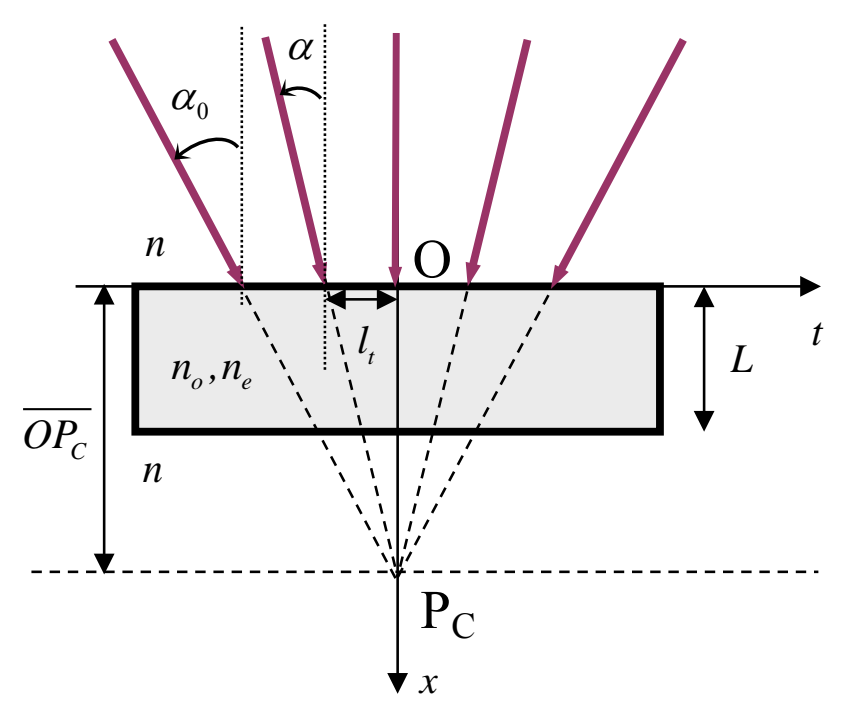

Fig. 6: Proyección sobre un plano de un haz cónico incidente sobre una placa plano-paralela.

Sobre el plano $\Pi$ los puntos de incidencia forman una circunferencia de radio

$$
r_{\Pi}^{\prime}=L \frac{n \sin \alpha}{\left(n_{o}^{2}-n^{2} \sin ^{2} \alpha\right)^{1 / 2}}+\left(D-\overline{O P_{C}}\right) \tan \alpha
$$

como resulta de las ecs. (10) y (42). Es decir, las coordenadas de los puntos de incidencia sobre el plano $\Pi$ de los rayos provenientes de la transmisión ordinaria son

$$
\begin{aligned}
& d_{y}^{\prime}=r_{\Pi}^{\prime} \sin \delta \\
& d_{z}^{\prime}=r_{\Pi}^{\prime} \cos \delta
\end{aligned}
$$

El punto de convergencia $D_{C}^{\prime}$ de estos rayos trasmitidos resulta de la ec.(45) considerando $r_{o}^{\prime}=0$

$$
D_{C}^{\prime}=\overline{O P_{C}}-L \frac{n \cos \alpha}{\left(n_{o}^{2}-n^{2} \sin ^{2} \alpha\right)^{1 / 2}}
$$

que es la misma expresión conocida para el punto de convergencia de la transmisión de una superficie cónica de rayos que atraviesa una placa plano-paralela isótropa. Y como $D_{C}^{\prime}$ depende de $\alpha$ resulta que, al igual que en las placas isótropas, los rayos que corresponden a un cono no se cruzan en un mismo punto.

Para los rayos extraordinarios, los puntos de incidencia en la segunda interfaz resultan de las ecs.(29) y (30)

$$
\begin{aligned}
l_{y}^{\prime \prime} & =\left(L \frac{n_{o} n \sin \alpha}{\left(n_{e}^{2} h_{x}-h_{\sigma} n^{2} \sin ^{2} \alpha\right)^{1 / 2}}-\overline{O P_{C}} \tan \alpha\right) \sin \delta \\
l_{z}^{\prime \prime}-A & =\left(L \frac{n_{e}^{2} n_{o} n \sin \alpha}{h_{x}\left(n_{e}^{2} h_{x}-h_{\sigma} n^{2} \sin ^{2} \alpha\right)^{1 / 2}}-\overline{O P_{C}} \tan \alpha\right) \cos \delta
\end{aligned}
$$

y sobre el plano $\Pi$ se obtiene, mediante ecs. (35) y (36)

$$
\begin{aligned}
& d_{y}^{\prime \prime}=\left(L \frac{n_{o} n \sin \alpha}{\left(n_{e}^{2} h_{x}-h_{\sigma} n^{2} \sin ^{2} \alpha\right)^{1 / 2}}+\left(D-\overline{O P_{C}}\right) \tan \alpha\right) \sin \delta(50) \\
& d_{z}^{\prime \prime}-A=\left(L \frac{n_{e}^{2} n_{o} n \sin \alpha}{h_{x}\left(n_{e}^{2} h_{x}-h_{\sigma} n^{2} \sin ^{2} \alpha\right)^{1 / 2}}+\left(D-\overline{O P_{C}}\right) \tan \alpha\right) \cos \delta
\end{aligned}
$$

Como estas ecuaciones dependen también del ángulo $\delta$, resulta que los rayos provenientes de una superficie cónica que inciden todos con el mismo ángulo $\alpha$ no se cruzan en un punto como resultó para los rayos ordinarios. De las ecs.(50) y (51) se obtienen las distancias $D_{C y}^{\prime \prime}$ y $D_{C z}^{\prime \prime}$ en los cuales se anulan $d_{y}^{\prime \prime}$ y $d_{z}^{\prime \prime}-A$. De esta manera

$$
\begin{gathered}
D\left(d_{y}^{\prime \prime}=0\right)=D_{C y}^{\prime \prime}=\overline{O P_{C}}-L \frac{n_{e} n_{o} n \cos \alpha}{h_{x}\left(h_{x}-n^{2} \sin ^{2} \alpha\right)^{1 / 2}} \\
D\left(d_{z}^{\prime \prime}=0\right)=D_{C z}^{\prime \prime}=\overline{O P_{C}}-L \frac{n_{o} n \cos \alpha}{\left(h_{x}\left(n_{e}^{2}-n^{2} \sin ^{2} \alpha\right)\right)^{1 / 2}}
\end{gathered}
$$

Consideraremos ahora, como ejemplo, un manojo de rayos dispuesto sobre una superficie cónica de ángulo $\alpha=30^{\circ}$ y cuyo vértice o punto de convergencia se encuentra a una distancia $\overline{O P_{C}}=10 \mathrm{~cm}$ de la placa. Los puntos de intersección de los rayos incidentes sobre la primera interfaz se encuentran sobre una circunferencia de radio $r=5.77 \mathrm{~cm}$. Los radios de las circunferencias sobre la segunda interfaz resultan de la ec.(43) $r^{\prime}=5.46 \mathrm{~cm}$ para la calcita y $r^{\prime}=5.58 \mathrm{~cm}$ para el rutilo, si el espesor de la placa es de $L=1 \mathrm{~cm}$. En estas condiciones, los respectivos puntos de convergencia (ec.(47)) se encuentran fuera de la placa a distancias 
$D_{C}^{\prime}=9.45 \mathrm{~cm} \quad$ y $D_{C}^{\prime}=9.66 \mathrm{~cm}$ para calcita $\mathrm{y}$ rutilo, respectivamente. Para la transmisión extraordinaria, el haz trasmitido no tiene simetría de revolución y aparece un astigmatismo de primer orden [12] a distancias de la placa dadas por las ecs. (52) y (53). Para la placa de calcita las posiciones de las imágenes astigmáticas están dadas por $D_{C_{y}}^{\prime \prime}=9.48 \mathrm{~cm} D_{C_{z}}^{\prime \prime}=9.37 \mathrm{~cm}$ y para la de rutilo por $D_{C_{y}}^{\prime \prime}=9.64 \mathrm{~cm} D_{C_{z}}^{\prime \prime}=9.70 \mathrm{~cm}$ correspondiendo la dirección del eje óptico a $\vartheta=25^{\circ}$ en ambas placas. En la Fig.7 se muestran los diagramas de puntos correspondientes a las figuras formadas a distintas distancias de la placa, que corresponden a las distancias de convergencia respectivas.
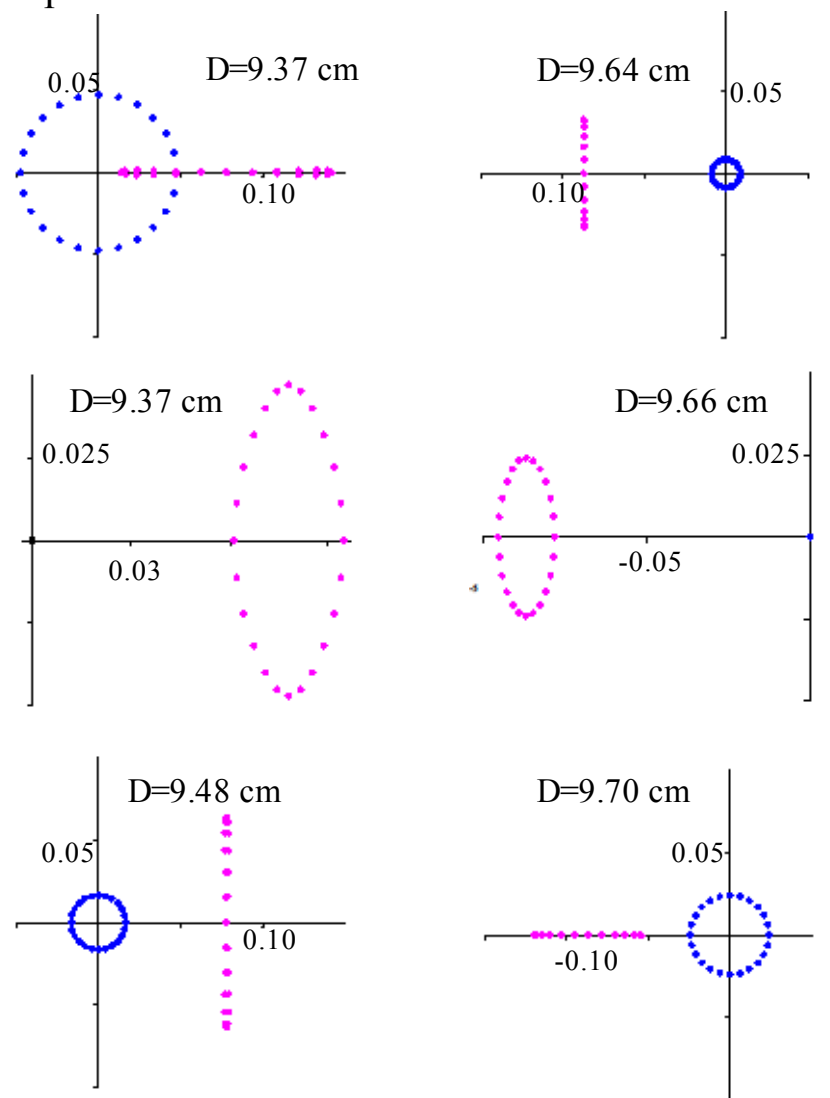

Fig. 7: Figuras que corresponden a las imágenes ordinaria y astigmática extraordinaria a través de una placa de calcita (izquierda) y una de rutilo (derecha) a distintas distancias de la cara inferior. Las distancias están presadas en centímetros. Los puntos azules corresponden a rayos ordinarios y los magenta a rayos extraordinarios.

En la Fig.8 se representan los diagramas de puntos (para un haz cónico de $30^{\circ}$ de apertura, con punto de convergencia $\overline{O P_{C}}=10 \mathrm{~cm}$ ) sobre la segunda interfaz y sobre un plano intermedio entre los planos que corresponden a las imágenes astigmáticas. Vemos que, sobre la segunda interfaz, ambos haces están superpuestos pero quedan separados en la zona en la cual se forman las imágenes que distan de ser figuras sencillas en el caso extraordinario.

En un trabajo anterior mostramos que cuando incide un haz de rayos plano convergente y en forma de abanico en un punto sobre la primera interfaz, los rayos refractados extraordinarios están todos contenidos en un plano que habíamos denominado plano de refracción [23]. Si consideramos, ahora, un haz cónico con el punto de convergencia en la primera interfaz, entonces, a la superficie cónica de ángulo $\alpha$ le corresponde una elipse en la segunda interfaz, como surge de las ecs.(48) y (49) para $\overline{O P_{C}}=0$. Se obtiene así la ecuación de una elipse con semiejes en las direcciones $y$ y $z$

$$
\frac{l_{y}^{\prime \prime 2}}{a^{2}}+\frac{\left(l_{z}^{\prime \prime}-A\right)^{2}}{b^{2}}=1
$$

donde

$$
\begin{gathered}
a=\frac{L n n_{o} \sin \alpha}{h_{x}^{1 / 2}\left(n_{e}^{2}-n^{2} \sin ^{2} \alpha\right)^{1 / 2}} \\
b=\frac{L n n_{o} n_{e} \sin \alpha}{h_{x}\left(h_{x}-n^{2} \sin ^{2} \alpha\right)^{1 / 2}}
\end{gathered}
$$

El tamaño de los semiejes depende no sólo de los índices de refracción de los medios y de la apertura del cono sino también de la dirección del eje óptico de la placa. Sin embargo, el tipo de elipse depende únicamente de la birrefringencia: cuando la birrefringencia es positiva, el eje mayor de la elipse está en la dirección del eje óptico sobre la interfaz (eje $z$ ) mientras que si es negativa está en la dirección perpendicular (eje y).

De todas maneras, bajo estas condiciones, el diagrama de puntos sobre cualquier plano $\Pi$ resulta no elíptico. Sin embargo, puede obtenerse una figura elíptica a la salida de la placa ajustando el plano $\Pi$ adecuadamente. En efecto, como surge de las ecs.(50) y (51), si la distancia de la placa al plano $\Pi$ se hace igual a la distancia entre la primera interfaz y el punto de convergencia del haz incidente (convergencia virtual), se obtendrá un diagrama de puntos de forma elíptica dado por

$$
\frac{d_{y}^{\prime \prime 2}}{a^{2}}+\frac{\left(d_{z}^{\prime \prime}-A\right)^{2}}{b^{2}}=1
$$

con semiejes dados por las ecs. (55) y (56). 


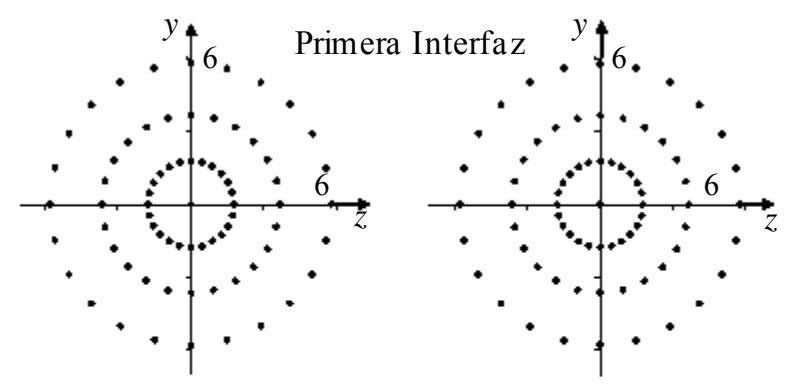

cilíndricos también es de interés especial porque corresponden a una aproximación de primer orden de los haces colimados.

Para un haz cónico con incidencia media normal a la placa, la pérdida de la simetría de revolución no es sólo del conjunto sino del haz extraordinario. Es decir, no sólo aparece el desplazamiento lateral sino una deformación asimétrica del haz mismo que da lugar a la imagen astigmática. Los resultados obtenidos para haces cónicos tridimensionales son de particular interés para el diseño de sistemas ópticos que combinen distintos elementos a lo largo del recorrido de la luz como moduladores y demoduladores birrefringentes.

\section{AgRADECIMIENTOS}

Queremos agradecer a la Dra. María C. Simon por sus valiosos aportes. Este trabajo fue realizado con el apoyo parcial de los siguientes subsidios

20020160100042BA- UBACYT 2017-2020

20020150200143BA- UBACYT 2016-2017

20020170200232BA -UBACYT 2018-2019

PICT $2016 \mathrm{~N}^{\circ} 2204$

\section{REFERENCIAS}

Fig. 8: Figuras que corresponden a las imágenes ordinarias y extraordinarias a través de una placa de calcite (izquierda) y de rutilo (derecha) para un haz cónico de rayos. Los puntos azules corresponden a los rayos ordinarios y los magenta a los extraordinarios.

\section{CONCLUSIONES}

De los cálculos efectuados podemos obtener una descripción cualitativa de las deformaciones de los haces de luz cilíndricos y cónicos cuando inciden sobre una placa plano-paralela uniaxial y la dirección de incidencia media es perpendicular a la misma. Los tipos de manojos de rayos analizados fueron elegidos con el propósito de poder distinguir qué características de los haces reales son las responsables de los distintos tipos de deformaciones geométricas.

Para el caso más sencillo, que es un haz cilíndrico, vimos que los dos haces transmitidos tienen la misma forma y tamaño que el haz incidente, pero el haz extraordinario queda desplazado con respecto al haz ordinario en una distancia y sentido que depende de la birrefringencia $n_{e}-n_{o}$ y de la dirección del eje óptico. Esto ya introduce una pérdida de simetría de revolución del conjunto de ambos haces aunque cada haz transmitido sea de contorno cilíndrico. El comportamiento de los haces

[1] E. Hecht y A. Zajac, Óptica, Fondo Educativo Interamericano, S.A., 1977

[2] M. C. Simon, "Ray tracing formulas for monoaxial optical components," Appl. Opt. 22, 354-360 (1983)

[3] M. C. Simon and R. M. Echarri, "Ray tracing formulas for monoaxial optical components: vectorial formulation," Appl. Opt. 25, 1935-1939 (1986)

[4] E. Cojocaru, "Direction cosines and vectorial relations for extraordinary-wave propagation in uniaxial media," Appl. Opt. 36, 302-306 (1997)

[5] O. N. Stavroudis, "Ray-tracing formulas for uniaxial crystals," J. Opt. Soc. Am.52, 187-191 (1962)

[6] J. D. Trollinger, R. A. Chipman and D. K. Wilson, "Polarization ray tracing in birefringent media," Opt. Eng. 30, 461-466 (1991)

[7] Simon, María C., and Karin V. Gottschalk. "Symmetries and asymmetries of the refracted and reflected rays in a uniaxial plane parallel plate." Optik-International Journal for Light and Electron Optics 123.1 (2012): 73-84.

[8] M. C. Simon and K. V. Gottschalk, "Optical path in birefringent media and Fermat's principle," Pure and Appl. Opt. Part A (JEOS) 7, 1403-1410 (1998)

[9] M. Avendaño-Alejo and M. Rosete-Aguilar, "Optical path difference in a plane-parallel uniaxial plate," J. Opt. Soc. Am. A 23, 926-932 (2006)

[10] M. C. Simon, M. T. Garea, "Plane parallel birefringent plates as polarization interferometers," Optik 87, 95-102 (1991)

[11] M. T. Garea and M. C. Duplaá, "Plane parallel biaxial plates as polarization interferometers," Optik 117, 321-328 (2006)

[12] F. E. Veiras, "Errores de Primer Orden En Láminas Retardadoras a Frecuencias Ópticas". Tesis de grado en Ingeniería Electrónica. (2008) Facultad de Ingeniería UBA.

[13] Veiras, Francisco E., Liliana I. Perez, and María T. Garea. "Phase shift formulas in uniaxial media: an application to waveplates." Applied Optics 49.15 (2010): 2769-2777.

[14] Uribe-Patarroyo, N., et al. "Space-qualified liquid-crystal variable retarders for wide-field-of-view coronagraphs." Solar Physics and Space Weather Instrumentation IV. Vol. 8148. International Society for Optics and Photonics, 2011. 
[15] Thorman, A., et al. "A photoelastic-modulator-based motional Stark effect polarimeter for ITER that is insensitive to polarized broadband background reflections." Review of Scientific Instruments 87.7 (2016): 073504.

[16] Samuell, C. M., et al. "Absolute calibration of Doppler coherence imaging velocity images." Journal of Instrumentation 12.08 (2017): C08016.

[17] Veiras, Francisco E., María T. Garea, and Liliana I. Perez. "Wide angle conoscopic interference patterns in uniaxial crystals." Applied optics 51.15 (2012): 3081-3090.

[18] Riobó, Lucas M., et al. "Interferómetro de polarización para la caracterización mecánica de dispositivos piezoeléctricos." Biennial Congress of Argentina (ARGENCON), 2014 IEEE. IEEE, 2014.

[19] Veiras, Francisco Ezequiel, et al. "Birefringent phase demodulator: application to wave plate characterization." Applied optics 54.9 (2015): 2326-2333.

[20] Veiras, F. E., M. T. Garea, and L. I. Perez. "A birefringent polarization modulator: Application to phase measurement in conoscopic interference patterns." Review of Scientific Instruments 87.4 (2016): 043113.

[21] M. C. Simon, "Image formation through monoaxial plane parallel plates," Appl. Opt. 27, 4176-4182 (1988)

[22] M. C. Simon and K. V. Gottschalk, "Waves and rays in uniaxial birefringent crystals," Optik 118, 457-470 (2007)

[23] Simon, María C., Liliana I. Perez, and Francisco E. Veiras. "Parallel beams and fans of rays in uniaxial crystals." AIP Conference Proceedings. Vol. 992. No. 1. AIP, 2008. 